\title{
An Analytical Solution for Fiber Optic Links with Photonic-Assisted Millimeter Wave Upconversion Due to MZM Nonlinearities
}

\author{
Romildo Henrique de Souza, Olympio Lucchini Coutinho, José Edimar Barbosa Oliveira \\ Instituto Tecnológico de Aeronáutica - ITA, São José dos Campos - SP, Brazil, \\ romildo@ita.br,olympio@ita.br,edimar@ita.br
}

Antônio Alves Ferreira Júnior, José Antônio Justino Ribeiro

Instituto Nacional de Telecomunicações - INATEL, Santa Rita do Sapucaí-MG, Brazil, antonioa@inatel.br, justino@inatel.br

\begin{abstract}
Photonic-assisted technique employed in the generation of stable and high-purity signals in the millimeter wave band has been investigated using dual-parallel Mach-Zehnder modulator. Indeed, this type of generation performs a high order RF multiplier, whose operation principle steams out from the nonlinear characteristic of the external modulators. Its application in directdetection fiber optic link was investigated based on an exact formulation that properly takes into account both the dispersion of a single mode fiber and the modulator nonlinearity. Analytic expression for the link transfer function dependence with respect to both modulator bias point and modulation index were obtained. It is worthwhile to mention that RF tuning is enabled since no optical filtering is required. As an illustration of the modeling usefulness, it is analytically shown that a $60 \mathrm{GHz}$ millimeter signal, generated by photonic quadruplicating a $15 \mathrm{GHz}$ signal using dual-parallel Mach-Zehnder modulator, can be transmitted through a $80 \mathrm{~km}$ link of standard single mode fiber with less than $0.1 \mathrm{~dB}$ deviation with respect to ideal flat response. This performance is much better than previously published results.
\end{abstract}

Index Terms - Chromatic Dispersion, Electro-optic Up-conversion, Fiber Optic, Radio over Fiber.

\section{INTRODUCTION}

End-user modern applications are requiring faster transmission rates and better signal stability, which could be mapped as improvements on network systems with wide bandwidth and low signal distortion. Increasing convergence in wireless services suggests a migration to millimeter wave spectrum, resulting on smaller antennas and greater beam directivity. These are essential requirements in on-board radar systems and satellite communication links, where size and weight are precious resources. Pure electronic signal generation in microwave and millimeter wave (MMW) bands is a complicated and relatively expensive process due to factors such as the complexity of the electronic modules, the extremely small size of some components, the high losses by propagation in wired lines or wave-guides, and the difficulty in isolating circuits from electromagnetic interference [1]. Low distortion, high stability and low phase noise are desirable characteristics in a carrier signal used in 
wireless networks and radar systems for electronic warfare [2], for instance.

Photonic approaches could be employed to generate high frequency carrier signal with such criteria by up-conversion of lower frequency reference signals available from electronic sources. Furthermore, photonic techniques offer tunability and relatively lower cost compared to purely electronic systems [3]. Additionally, a fiber optic link allows providing the signal to a remotely located site before reconversion into the electrical domain, resulting in integration between optical processing and transmission (Fig. 1).

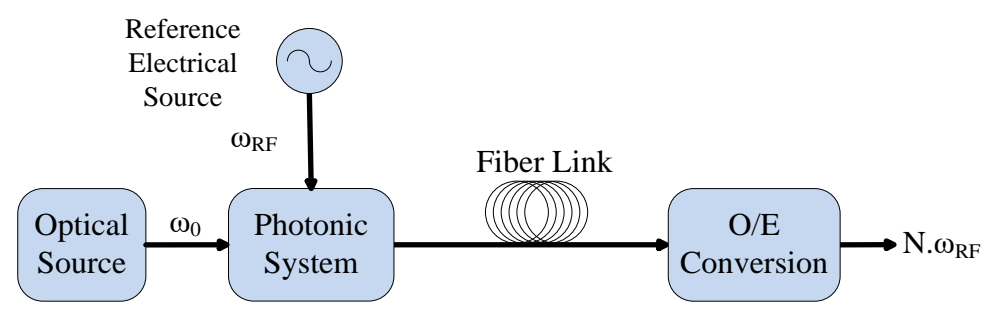

Fig. 1. Schematic representation of a general photonic-assisted frequency multiplication system used in remote applications (O/E - Optical/Electrical).

Among several proposed photonic-assisted frequency multiplication systems, those using MachZehnder Modulators (MZM) reveal being a convenient and scalable solution, whose operation principle steams out from the nonlinear characteristic of the external modulators. Researches for obtaining sixteen-fold frequency signals, with $20 \mathrm{~dB}$ of Sideband Suppression Ratio (SSR), represent state of the art in this area [4]. Examples of experimental demonstrations include an octupler at 42.4 $\mathrm{GHz}$, with electric SSR greater than $30 \mathrm{~dB}$ [5], and a quadrupler at $72 \mathrm{GHz}$ with $36 \mathrm{~dB}$ of optical SSR [6].

Dual Parallel MZM (DP-MZM) topologies do not require optical filtering, since parameters are properly settled in order to suppress optical carrier and certain harmonics. Commercial availability of devices with two or more modulators encapsulated in the same package opens the possibilities to explore different configurations. For example, consider two push-pull modulators in parallel and then connected to a third MZM as presented in Fig. 2a. Two electrical signals in quadrature are applied each one to a modulator, which are both biased at the Maximum Transmission Bias Point (MATB), while the third MZM operates at Minimum Transmission Bias Point (MITB) [6], [7]. An alternative topology (Fig. 2b) employs two dual drive modulators in parallel, eliminating the use of a third cascade MZM [8]. Both modulators are biased at MITB and the electric input signals on the same modulator are in quadrature, while $\pi$ out-of-phase from the other modulator. However, analysis of these topologies have been performed by using approximate methods restricted to small signal regime, where modulation index were arbitrarily limited to low values [6], [7], [8]. 
(a)
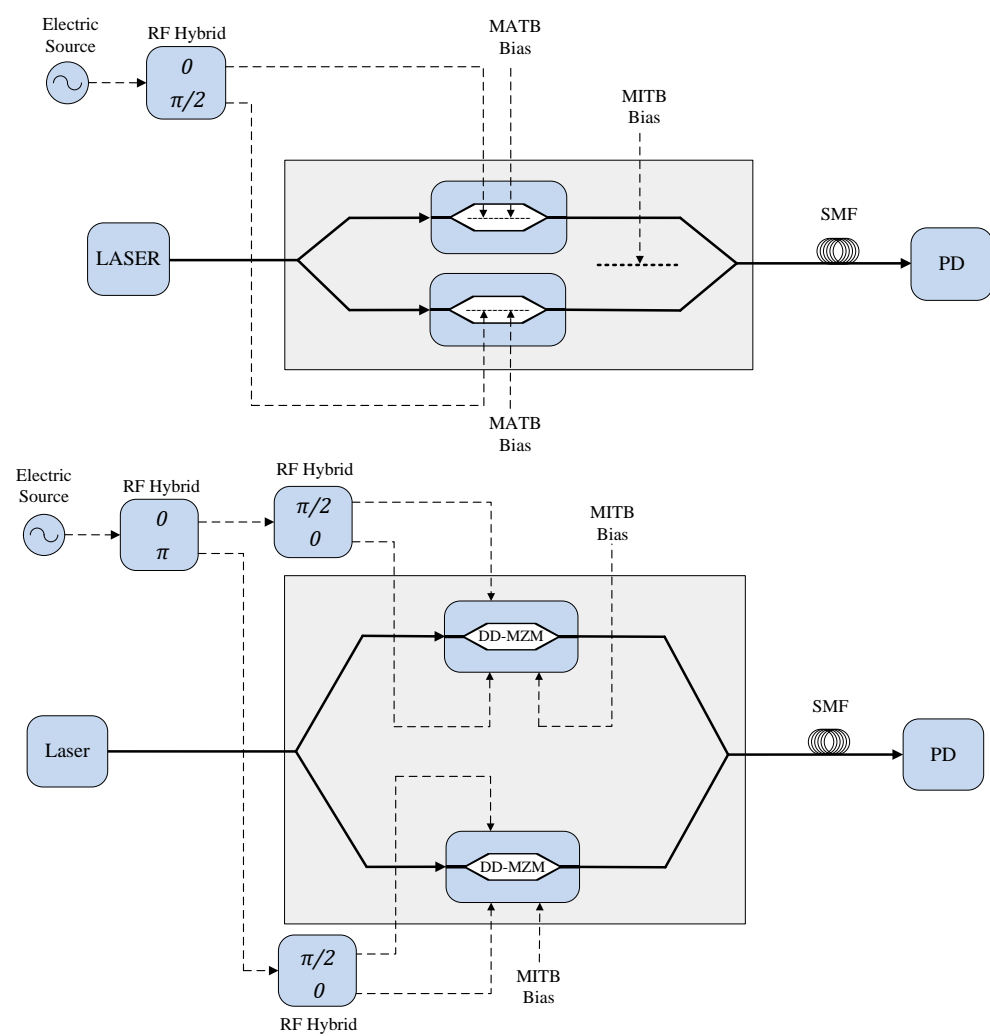

Fig. 2. Photonic-assisted frequency multiplication topologies based on: (a) push-pull MZM in parallel; (b) dual-drive MZM in parallel.

This paper is mainly concerned with the modeling of the effect of the MZM nonlinearity on the performance of optical up-conversion. Special attention is given to the MMW generation which relies on Dual-Parallel MZM (DP-MZM) and exact expressions for the harmonic amplitude dependence on both modulation index and bias point were deduced [9]. Based on such formulation we were able to precisely predict the harmonic distortion suppression ratio within a range of optical modulation index far wider than that used in previous publications [6], [7], [8]. Additionally, a fiber optic spool is inserted on the modulator output for remote transmission. Under such conditions, chromatic dispersion influences system multiplication factor and thus must be considered on the final project design [10].

At this scope, the fundamentals of optical up-conversion are presented in Section II, which addresses the optical and RF spectra at the output of a Dual-Drive Mach-Zehnder Modulator (DDMZM) at distinct bias point conditions. Section III deduces the wave propagation equation in fiber optic links under chromatic dispersion and attenuation effects and relates them with a properly transfer function to account amplitude and phase response for each optical spectral component. A DDMZM combined with a spool of fiber is the simplest frequency multiplication topology, which is rigorous analyzed in Section IV, aiming to establish the exact solution methodology in order to realize the effect of fiber chromatic dispersion on the multiplication factor. Section $\mathrm{V}$ applies the formulation on a dual parallel MZM scenario, resulting in higher multiplication factors and consequently reaching millimeter wave generation. Thus, this exact solution is applied to a back-to-back (BTB) system in 
Section VI, in order to compare with approximated solution found in the literature. Finally, conclusion and brief discussion are presented in Section VII.

\section{FUNDAMENTALS OF DD-MZM UP-CONVERTER}

Although the field of electro-optic modulation is well established, its application for accomplishing optical MMW signal generation, in which the MZM works as a frequency multiplier, had begun rather recently. Therefore, in order to be able to properly model such device, we consider worthwhile to present a brief review of the main characteristics of DD-MZM's operation. To this end, the MZM electronic drives should be assembled in such a way to enable its operation at any desired bias point on its transference curve. Then, the DD-MZM will be part of a fiber optic link, as depicted in Fig. 3. The operation of such type of modulator relies on the interference between two optical guided modes whose propagation constant are controlled by a RF electric field pattern conveniently distributed inside an electro-optic substrate. The modulating electric field pattern, which is spatially formatted by thin film electrodes, behaves as quasi-TEM wave. It depends on RF reflection coefficients at the ends of the electrodes and on the optical and RF velocities mismatching (see Appendix for further detail). The field pattern is driven by a combination of DC and RF sources, which provides the bias and RF modulating field, respectively. In this publication, it is assumed that the impedance and velocity matching are simultaneously attained, hence the RF phase shift induced by the voltage waveforms seen by the optical wave is given by (A-6). Under such condition, the RF inputs of the DD-MZM shown in Fig. 3 are specified by:

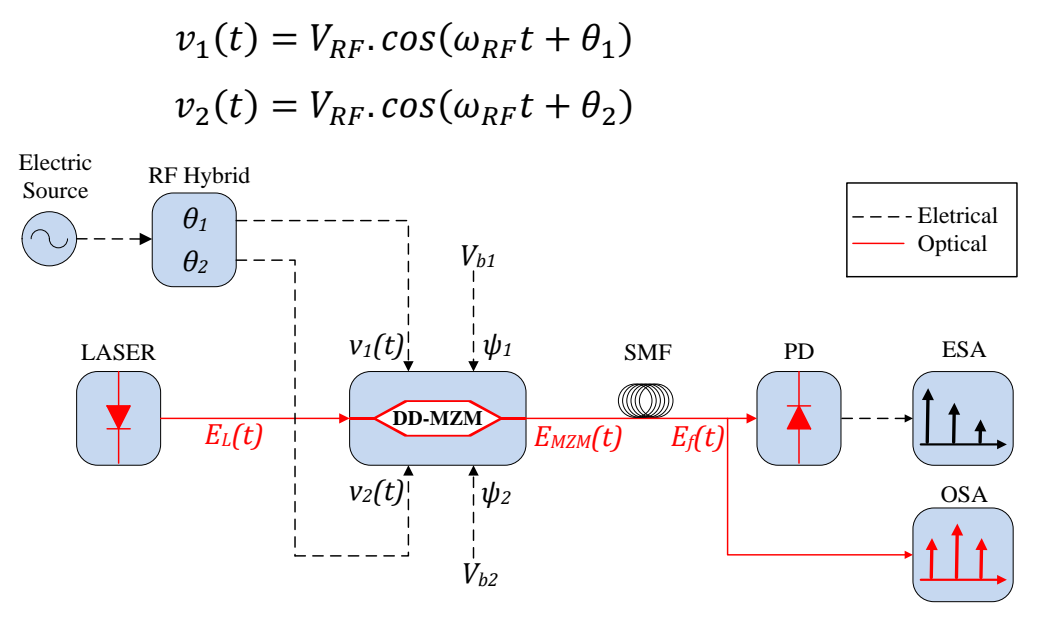

Fig. 3. Schematic representation of a fiber optic link based on intensity modulation and direct detection (DD-MZM: Dual Drive Mach-Zehnder Modulator; SMF: Single Mode Fiber; PD: Photodetector; OSA: Optical Spectrum Analyzer; ESA: Electrical Spectrum Analyzer).

With respect to the optical carrier shown in Fig. 3, it is assumed that it steams out from a DFB laser with null linewidth and no intensity or phase noise, which yields an optical power $P_{0}$ and corresponding electrical field strength $E_{0}$ and frequency $\omega_{0}$.

Assuming a 50/50 splitting ratio and no insertion loss on the modulator, following rather standard procedures, the electric optical field at the DD-MZM output is obtained as: 


$$
E_{M Z M}^{D D}(t)=E_{0} A_{I M} e^{j \omega_{0} t} e^{j \varphi_{P M}}
$$

where

$$
\begin{gathered}
A_{I M}=\cos \left[m \cdot \sin \left(\omega_{R F} t+\frac{\theta_{1}+\theta_{2}}{2}\right) \cdot \sin \left(\frac{\theta_{1}-\theta_{2}}{2}\right)-\left(\frac{\psi_{1}-\psi_{2}}{2}\right)\right] \\
\varphi_{P M}=m \cdot \cos \left(\omega_{R F} t+\frac{\theta_{1}+\theta_{2}}{2}\right) \cdot \cos \left(\frac{\theta_{1}-\theta_{2}}{2}\right)+\frac{\psi_{1}+\psi_{2}}{2} \\
m=\frac{\pi V_{R F}}{V_{\pi}^{R F}} \quad \psi_{1}=\frac{\pi V_{b 1}}{V_{\pi}^{D C}} \quad \psi_{2}=\frac{\pi V_{b 2}}{V_{\pi}^{D C}}
\end{gathered}
$$

being $m$ the optical modulation index; $V_{\pi}^{R F}$ and $V_{\pi}^{D C}$ the half-wave voltages, at RF frequency and DC, respectively; and, $V_{b 1}$ and $V_{b 2}$ the DC bias voltage.

An examination of (3), (4) and (5) reveals that, in general, the output signal has a combination of amplitude and phase modulation. However, when both RF phase shifts $\left(\theta_{1}\right.$ and $\left.\theta_{2}\right)$, and DC bias $\left(\psi_{1}\right.$ and $\psi_{2}$ ) are properly chosen, it is possible to achieve different modulation formats which ranges from pure intensity to pure phase modulation. Moreover these generalized expressions turns out to be very helpful when we undertake the modeling of a DP-MZM, which is made up of two adequately electronically driven DD-MZM's that are optically associated in a parallel configuration. Beyond that, bearing in mind that the DD-MZM's performance evaluation as an up-converter requires the knowledge of its spectra characteristics, i.e., both the optical and RF spectrum, the setup illustrated in Fig. 3 includes both an optical spectrum analyzer (OSA) and an electronic spectrum analyzer (ESA).

Using Jacobi-Anger expansion in (3), the electrical field can be represented as:

$$
E_{M Z M}^{D D}(t)=\frac{E_{0}}{2} \sum_{n=-\infty}^{\infty} a_{n} e^{j\left(\omega_{0}+n \omega_{R F}\right) t}
$$

with

$$
a_{n}=e^{j \frac{n \pi}{2}}\left[e^{j\left(n \theta_{1}+\psi_{1}\right)}+e^{j\left(n \theta_{2}+\psi_{2}\right)}\right] J_{n}(m)
$$

where $J_{n}(m)$ is the $n$-th Bessel function of first kind.

As previously stated, this section aims at the modeling of an electro-optical up-conversion based on DD-MZM, and validating the obtained results through their comparison to well accepted results available in the literature, whenever it is possible. To this end, we have to particularize the schematic depicted in Fig. 3, in a way that it reduces to well established configurations [11], [12]. Such references present valuable results regarding to both experimental and theoretical on the DD-MZM modulator performance when it operates either in single sideband (SSB) or double sideband (DSB) regime.

In order to be able to compare our results to those given in [12], we particularize the configuration shown in Fig. 3 by imposing $\theta_{2}=\psi_{2}=0$. Under these conditions, (4) and (5) become:

$$
A_{I M}=\cos \left[m \cdot \sin \left(\omega_{R F} t+\frac{\theta_{1}}{2}\right) \cdot \sin \left(\frac{\theta_{1}}{2}\right)-\frac{\psi_{1}}{2}\right]
$$




$$
\varphi_{P M}=m \cdot \cos \left(\omega_{R F} t+\frac{\theta_{1}}{2}\right) \cdot \cos \left(\frac{\theta_{1}}{2}\right)+\frac{\psi_{2}}{2}
$$

Furthermore, the above given equations provide the following expression for the optical power at the output of the DD-MZM, i.e., in the so-called back-to-back (BTB) situation:

$$
P_{M Z M}^{D D}(t)=P_{0} \cos ^{2}\left[m \cdot \sin \left(\omega_{R F} t+\frac{\theta_{1}}{2}\right) \cdot \sin \left(\frac{\theta_{1}}{2}\right)-\frac{\psi_{1}}{2}\right]
$$

Equation (11) besides revealing the nonlinear dependence of the DD-MZM response with respect to the driving electronic signal, also points out that it can be strongly affected by the bias point, which is specified by a combination of phase shift and DC bias, namely the pair $\left(\theta_{1}, \psi_{1}\right)$. In general, the literature emphasizes the conditions which yield maximum transmission (MATB), quadrature (QB) and minimum transmission (MITB). In this paper we are interested in those situations in which $\left(\theta_{1}, \psi_{1}\right)$ meet the following requirements: $(\pi, 0)$ for MATB; $(\pi, \pi / 2)$ for QB; and $(\pi, \pi)$ for MITB. The localizations of such points over the modulator characteristic curve are shown in Fig. 4.

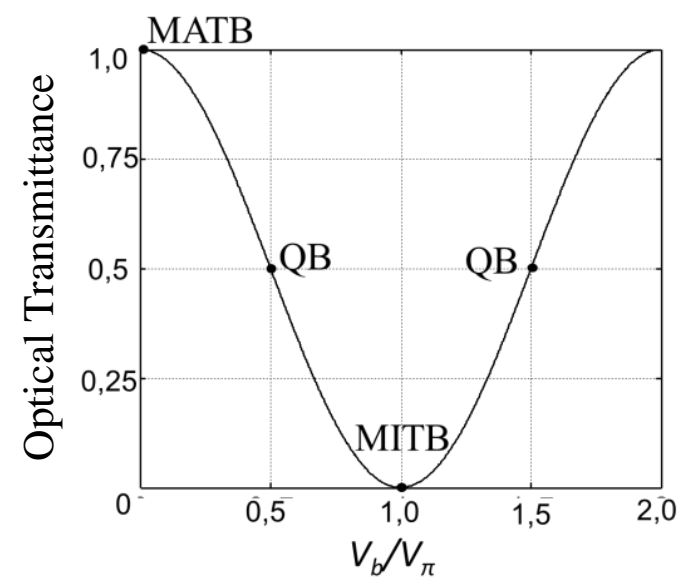

Fig. 4. DD-MZM static optic transmission characteristic curve.

Once again using the Jacobi-Anger expansion in (11), we obtain the optical power spectral component at frequency $\omega_{0}+n \omega_{R F}$ :

$$
P_{M Z M}^{D D}\left(\omega_{0}+n \omega_{R F}\right)=P_{0} \cdot J_{n}^{2}(m) \cdot \cos ^{2}\left(\frac{n \theta_{1}+\psi_{1}}{2}\right)
$$

The result given by (12) has been widely used in both theoretical and experimental investigations of high speed electro-optic modulators performance [11].

Before undertaking any further analysis, let us point out a few features of the dependence of the optical power spectrum with respect to a chosen bias point, as predicted by (12). First of all, one realizes that both the amplitude of a given optical spectral component and the spectrum symmetry with respect to the carrier can be controlled by properly choosing the bias point, defined by $\left(\theta_{1}, \psi_{1}\right)$, as shown in Fig. 3. As an illustration, we choose $\left(\theta_{1}, \psi_{1}\right)=(\pi / 2, \pi / 2)$, which implies that the $+1^{\text {st }}$ order sideband is null, whereas the $-1^{\text {st }}$ order sideband is given by $P_{0} J_{1}^{2}(m)$. Thereby, such bias point yields the so-called single sideband regime (SSB). A rather different feature is obtained when we choose $\left(\theta_{1}, \psi_{1}\right)=(\pi, 0)$, because the $\pm 1^{\text {st }}$ order sidebands, are suppressed while the remaining 
components are all separated by even multiple of the MMW frequency $\omega_{R F}$. This configuration is called double sideband (DSB) and its spectra, at both optical and RF domains, are depicted in Fig. 5.
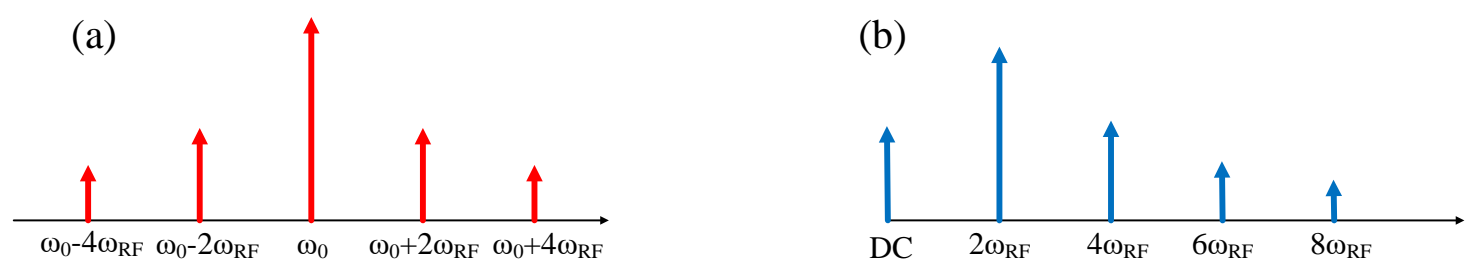

Fig. 5. Frequency response (amplitudes not in scale) of a DD-MZM operating at MATB in the DSB regime: (a) optical spectrum; (b) electronic spectrum.

Now, we return to (12) aiming to predict the electrical current at the output of a photodetector (PD), when the setup shown in Fig. 3 is rearranged to perform BTB measurement. Obviously, under such assumption the fiber optic chromatic dispersion is not taken into account, therefore the MMW waveform and its corresponding spectral composition can be readily determined based on (12). One concludes that the electric current waveform is given by the following expression:

$$
I_{M Z M}^{D D}(t)=\Re P_{0} \cos ^{2}\left[m \cdot \sin \left(\omega_{R F} t+\frac{\theta_{1}}{2}\right) \cdot \sin \left(\frac{\theta_{1}}{2}\right)-\frac{\psi_{1}}{2}\right]
$$

where $\mathfrak{R}$ is the PD responsitivity.

In previous publication we have used the Jacobi-Anger expansion in (13) and then carried out a general analysis of the dependence of electrical signal spectral content, at the output of a DD-MZM, with respect to the bias point, i.e., $\left(\theta_{1}, \psi_{1}\right)$ [13]. Such undertaking, beyond enabling a quick retrieve of a wide range of results available in the literature regarding to the single tone operation of DDMZM, also provided valuable insights on the subject of bias control techniques for lithium niobate modulators [13]. Part of the obtained results which are concerned to the optical and electronic power spectra with the DD-MZM operating in the DSB regime are given in Table I. This type of configuration played a major role during the earliest years of optical research on microwave optical generation by electro-optic up-conversion [14]. It is worthwhile to point out that (13) can be rewritten in the rather familiar expression shown below:

$$
I_{M Z M}^{D D}(t)=\Re \frac{P_{0}}{2}\left\{1+\cos \left[2 m \cdot \sin \left(\omega_{R F} t+\frac{\theta_{1}}{2}\right) \cdot \sin \left(\frac{\theta_{1}}{2}\right)-\psi_{1}\right]\right\}
$$

which provides a manner for quick double-checking of all predictions so far presented in this publication.

TABLE I. OPTICAL AND ELECTRONIC POWER SPECTRAL AMPLITUDE FOR MATB, QB, MITB (DSB WITH $\left.\theta_{1}=\pi\right)$

\begin{tabular}{ccccc}
\hline Bias & \multicolumn{2}{c}{ Normalized Optical Power } & \multicolumn{2}{c}{ Normalized Electrical Power } \\
\cline { 2 - 5 } Point & n even & n odd & n even & n odd \\
\hline MATB & $J_{n}^{2}(m)$ & 0 & $\frac{J_{n}^{2}(2 m)}{2}$ & 0 \\
QB & $\frac{J_{n}^{2}(m)}{2}$ & $\frac{J_{n}^{2}(m)}{2}$ & 0 & $\frac{J_{n}^{2}(2 m)}{2}$
\end{tabular}




\begin{tabular}{ccccc}
\hline \multirow{2}{*}{$\begin{array}{c}\text { Bias } \\
\text { Point }\end{array}$} & \multicolumn{2}{c}{ Normalized Optical Power } & \multicolumn{2}{c}{ Normalized Electrical Power } \\
\cline { 2 - 5 } MITB & 0 & $J_{n}^{2}(m)$ & $\frac{J_{n}^{2}(2 m)}{2}$ & n odd \\
\hline \multirow{2}{*}{ MITen } & 0 & & 0 \\
\hline
\end{tabular}

\section{CHROMATIC DISPERSION IN FIBER OPTIC ANALOG LINKS}

Optical signal transmitted through single mode fiber is influenced by some effects like silica attenuation, distortion caused by chromatic dispersion and spurious due to fiber nonlinear effects. Such features will modify the resulting electrical signal at the photodetector output and decrease system dynamic range. Once physical properties of dispersive materials depend on the transmitted signal frequency, phase factor $\beta(\omega)$ can be expanded in Taylor series around optical carrier angular frequency $\omega_{0}[15]$ :

$$
\beta(\omega)=\beta\left(\omega_{0}\right)+\left.\sum_{k=1}^{\infty}\left(\omega-\omega_{0}\right)^{k} \frac{1}{k !} \frac{\partial^{k} \beta}{\partial \omega^{k}}\right|_{\omega=\omega_{0}}
$$

Relying on slowly varying envelope approximation, the solution of the electric field wave equation assuming z-propagation and $\mathrm{x}$-linear polarization is [15]:

$$
\vec{E}(\vec{r}, t)=F\left(x, y, \omega_{0}\right) \cdot A(z, t) \cdot e^{j\left[\beta\left(\omega_{0}\right) z-\omega_{0} t\right]} \hat{x}
$$

where $F\left(x, y, \omega_{0}\right)$ is the electrical field spatial distribution at frequency $\omega_{0}$ and $A(z, t)$ is the slowly varying wave envelope.

Plugging (16) in the wave equation and using the expanded phase factor $\beta(\omega)$, given by (15), first order perturbation theory leads to [15]:

$$
\frac{\partial A}{\partial z}+\frac{\alpha_{t}}{2} A=j \sum_{k=1}^{\infty} \frac{(j)^{k} \beta_{k}}{k !} \frac{\partial^{k} A}{\partial t^{k}}+j \gamma \cdot\left(1+\frac{j}{\omega_{0}} \frac{\partial}{\partial t}\right) \cdot\left[A(z, t) \cdot \int_{0}^{\infty} R\left(t^{\prime}\right) \cdot\left|A\left(z, t-t^{\prime}\right)\right|^{2} d t^{\prime}\right]
$$

where $\beta_{k}=\left.\frac{\partial^{k} \beta}{\partial \omega^{k}}\right|_{\omega=\omega_{0}}$ are the phase factor coefficients, $\alpha_{t}$ is the attenuation factor, $\gamma$ is the nonlinear parameter due to Kerr effect and $R(t)$ is the nonlinear response function due to Raman scattering, considering it as the most intense nonlinear phenomena in that case.

Let us assume intensity modulation of an optical carrier with angular frequency $\omega_{0}$ by an electric signal $\omega_{R F}$, being transmitted through a fiber optic link. The electric field spectral components of the optical signal will be at frequencies $\omega_{0} \pm n \omega_{R F}$, for any integer $n$, in a DSB modulation. Without losing accuracy and following general approach, the power series in (15) could be limited until the third term to obtain a reasonable phase factor for each spectrum component:

$$
\beta\left(\omega_{0}+n \omega_{R F}\right)=\beta_{0}+n \omega_{R F} \beta_{1}+\frac{1}{2}\left(n \omega_{R F}\right)^{2} \beta_{2}
$$

where $\beta_{0}=\beta\left(\omega_{0}\right)$ gives rise to a fixed phase shift; $\beta_{1}=\left.\frac{\partial \beta}{\partial \omega}\right|_{\omega=\omega_{0}}$ is the inverse of the group velocity, which makes the second term in (18) be linear in frequency and consequently results on a fixed 
propagation delay time; $\beta_{2}=\left.\frac{\partial^{2} \beta}{\partial \omega^{2}}\right|_{\omega=\omega_{0}}$ is related to the fiber linear dispersion parameter $D=$ $-\frac{2 \pi c}{\lambda^{2}} \beta_{2}\left(\omega_{0}\right)$, so third term leads to a linear propagation delay in frequency. It results on a distortion in the final waveform.

Neglecting fiber nonlinearities, once it is out of the scope of this paper, we set $\gamma=0$ in (17):

$$
\frac{\partial A}{\partial z}+\frac{\alpha_{t}}{2} A+\beta_{1} \frac{\partial A}{\partial t}+j \frac{\beta_{2}}{2} \frac{\partial^{2} A}{\partial t^{2}}=0
$$

As $\beta_{1}$ relates to a constant propagation delay, it turns to be convenient to apply the following transformation of variables [15], $T=t-\beta_{1} z$ and $Z=z$, in order to simplify (19):

$$
\frac{\partial A}{\partial Z}+\frac{\alpha_{t}}{2} A+j \frac{\beta_{2}}{2} \frac{\partial^{2} A}{\partial T^{2}}=0
$$

The above equation describes electromagnetic wave propagation in fiber optic media and could be denoted by a transfer function $H(\omega)$ for a spool of fiber with length $z=L$. Each spectral component has different propagation delays, i.e., phase factor depends on the component frequency, which leads to distinct phases for each component. Thus, transfer function $H(\omega)$ is defined by an amplitude response $U(\omega)$ and by a phase response $\phi(\omega)$ :

$$
H(\omega)=U(\omega) e^{j \phi(\omega)}
$$

Although silica attenuation varies with signal frequency, here it will be assumed as a constant inside the $1550 \mathrm{~nm}$ optical transmission window. Doing so, amplitude response $U(\omega)$ becomes:

$$
U=e^{\frac{\alpha_{t}}{2} L}=10^{\frac{-\alpha_{d B} L}{20}}
$$

where $\alpha_{d B}$ is the fiber attenuation factor in $\mathrm{dB} / \mathrm{km}$.

On the other hand, phase response for each optical spectral component of the modulated signal after propagation through a $L$-length fiber is:

$$
\phi\left(\omega_{0}+n \omega_{R F}\right)=\frac{1}{2}\left(n \omega_{R F}\right)^{2} \beta_{2}\left(\omega_{0}\right) L
$$

It could be noted that there is a quadratic relation between phase and frequency $f_{R F}$ for each component, $\phi_{n}=k_{n} f_{R F}^{2}$, where the proportionality factor $k_{n}$ is given by:

$$
k_{n}=2 \pi^{2} n^{2} \beta_{2}\left(\omega_{0}\right) L
$$

Rewriting (24) in terms of the linear dispersion parameter $D$, we have:

$$
k_{n}=-\pi n^{2} D \frac{\lambda_{0}^{2}}{c} L
$$

where $\lambda_{0}$ is the optical signal wavelength and $c$ is the speed of light.

On a direct detection receiver, quadratic photodetector output has a RF fundamental component which electrical power $P_{1}$ is proportional to $\cos ^{2}\left(k_{1} f_{R F}^{2}\right)$. This is null when argument is a $\pi /$ odd multiple [16]. Therefore, when a signal with frequency $f_{R F}$ is launched into a fiber optic link, the fundamental electric power component will ideally vanish at a first distance $L_{1}=\frac{c}{2 D \lambda_{0}^{2} f_{R F}^{2}}$ and then at 
each distance interval $\Delta L=\frac{c}{D \lambda_{0}^{2} f_{R F}^{2}}$. The electric power flows from fundamental component to higherorder harmonics and this phenomenon is known as signal fading [13], [17]. Fig. 6 presents a numerical example of the variation of the fundamental electrical power on the photodetector output when $30 \mathrm{GHz}$ and $60 \mathrm{GHz}$ signals are modulated by a $1550 \mathrm{~nm}$ optical carrier and transmitted through a standard single mode dispersive fiber $(D=17 \mathrm{ps} / \mathrm{nm}-\mathrm{km})$.

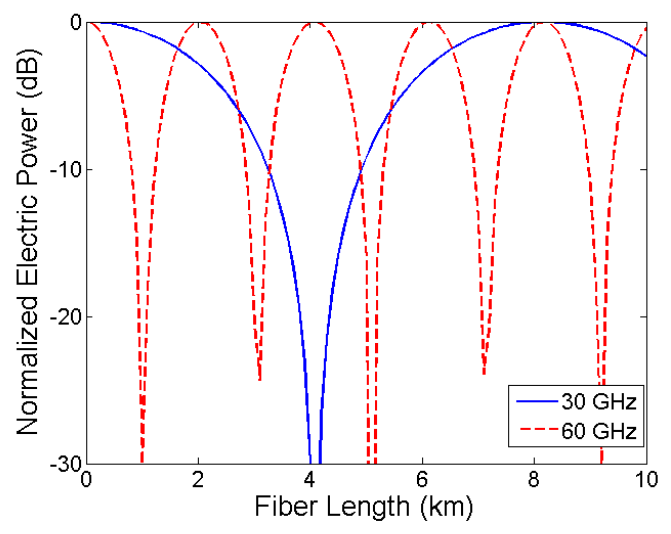

Fig. 6. Example of the fundamental electrical power dependence on fiber length for $30 \mathrm{GHz}$ and $60 \mathrm{GHz}$ signal due to chromatic dispersion effect on an intensity modulation/direct detection fiber optic link [17].

\section{MZM UP-CONVERSION IN FIBER OPTIC LINKS}

In order to develop the link model which takes into account the fiber chromatic dispersion, we should emphasize some assumptions we are taken for granted with respect to link setup shown in Fig. 3. First, as far as the optical signal is concerned, all components behave linearly, except the PD which displays quadratic response; secondly, the source of RF nonlinearity is fully concentrated into the DDMZM; and thirdly, the chromatic dispersion steams out from the optical fiber [11], [12]. Additionally, due to our interest in electro-optic up-conversion, it is convenient to DD-MZM to operate at MATB bias point.

Under the above mentioned assumptions, we used (7) and (8) to determine the optical electrical field in the time domain, right at the DD-MZM's output. Using this result in combination with Maxwell's equations, we undertook the time domain analysis of the propagation of the optical field along a dispersive and linear spool of SMF. In order to take into account the chromatic dispersion's effect, we will consider (21), (22) e (23) to find the electrical field at the optical link output:

$$
E_{f}(t)=10^{\frac{-\alpha_{d B} L}{20}} \frac{E_{0}}{2} \sum_{n=-\infty}^{\infty} a_{n} e^{j\left[\left(\omega_{0}+n \omega_{R F}\right) t+\phi\left(\omega_{0}+n \omega_{R F}\right)\right]}
$$

Assuming that the optical power is evenly distributed over the PD cross section, the time dependent RF current turns out to be:

$$
i_{P D}(t)=\frac{\Re\left[E_{f}(t)\right]\left[E_{f}(t)\right]^{*}}{2 Z_{o}}
$$

where $Z_{o}$ is a constant (in ohms per square meter) which depends on both the fiber effective crosssection and the optical wave impedance, and mathematical symbol * denotes the complex conjugate. 
Substituting (26) in (27), photodetector current becomes:

$$
i_{P D}(t)=10^{\frac{-\alpha_{d B} L}{10}} \frac{\Re E_{0}^{2}}{2 Z_{o}} \sum_{N=-\infty}^{\infty} i\left(N \omega_{R F}\right) e^{j N \omega_{R F} t}
$$

where each current harmonic component is:

$$
i\left(N \omega_{R F}\right)=\frac{1}{4} \sum_{n=-\infty}^{\infty} a_{n+N} a_{n}^{*} e^{j \phi_{n+N}} e^{-j \phi_{n}}
$$

for $\phi_{n}$ and $\phi_{n+N}$ being, respectively, the phase of components $n \omega_{R F}$ and $(n+N) \omega_{R F}$.

When we take these phases from (23), equation (29) becomes [13], [14]:

$$
i\left(N \omega_{R F}\right)=\frac{1}{4} e^{j N \frac{\Gamma}{2}} \sum_{n=-\infty}^{\infty} a_{n+N} a_{n}^{*} e^{j n \Gamma}
$$

with $\Gamma$ being a phase parameter due to fiber dispersion:

$$
\Gamma=N \beta_{2} \omega_{R F}^{2} L
$$

Taking $a_{n}$ coefficients from (8) and substituting them in (30), we apply Graf's Addition Theorem [12] to obtain [10]:

$$
\begin{aligned}
i\left(N \omega_{R F}\right)=\frac{1}{4}\{ & e^{j N \pi}\left(e^{j N \theta_{1}}+e^{j N \theta_{2}}\right) J_{N}\left[2 m \cdot \sin \left(\frac{\Gamma}{2}\right)\right] \\
+ & e^{-j \frac{N \Delta \theta}{2}} e^{j \Delta \psi} e^{j N \theta_{1}} e^{j N \pi} J_{N}\left[2 m \cdot \sin \left(\frac{\Gamma-\Delta \theta}{2}\right)\right] \\
+ & \left.e^{j \frac{N \Delta \theta}{2}} e^{-j \Delta \psi} e^{j N \theta_{2}} e^{j N \pi} J_{N}\left[2 m \cdot \sin \left(\frac{\Gamma+\Delta \theta}{2}\right)\right]\right\}
\end{aligned}
$$

where $\Delta \theta=\theta_{1}-\theta_{2}$ and $\Delta \psi=\psi_{1}-\psi_{2}$.

Clearly, harmonic electric current components depend on $\Gamma$ parameter and modulation index $m$, which are function of optical fiber parameters (length and dispersion coefficient), modulator halfwave voltage and bias voltage, and RF signal characteristics (amplitude, frequency and phases). Laser power, photodetector responsitivity and fiber attenuation are common terms for all the harmonics. Dominant harmonic and the relative intensity to the other harmonics are defined by this set of parameters. Adequate scenarios give rise to an output signal which frequency is a multiple of the reference signal frequency.

For DSB modulation ( $\left.\Delta \theta=\theta_{1}=\pi, \Delta \psi=\psi_{1}, \theta_{2}=\psi_{2}=0\right)$, (32) simplify to [9]:

$$
i\left(N \omega_{R F}\right)=\frac{1}{4}\left\{\left(1+e^{j N \pi}\right) J_{N}\left[2 m \cdot \sin \left(\frac{\Gamma}{2}\right)\right]+\left(e^{j \psi_{1}} e^{j \frac{N \pi}{2}}+e^{-j \psi_{1}} e^{-j \frac{N \pi}{2}}\right) J_{N}\left[2 m \cdot \cos \left(\frac{\Gamma}{2}\right)\right]\right\}
$$

In this case, there are only even harmonics and the lowest order multiplier is a doubling. Fig. 7a e $7 \mathrm{~b}$ show the relative RF power for second and fourth harmonics as a function of both $\Gamma$ parameter and modulation index $m$, for DSB modulation and MATB operation. Given a modulation index, it is possible to analyze system performance in terms of $\Gamma$ parameter by cutting the 3D-graphs, as done in Fig. $8 \mathrm{a}$ and $8 \mathrm{~b}$, for $m=1$ and $m=2.5$, respectively. On the first cut-plot, second harmonic is more intense than the fourth in a large $\Gamma$ interval and the difference between them reaches $20 \mathrm{~dB}$. However, 
specific $\Gamma$ values cause second harmonic to fade and the fourth harmonic dominates during a short interval, where instabilities do not ensure a good operation as a quadrupling. In order to improve this sensibility, modulation index could be increased. For instance, when $m=2.5$ the cut-plot shows that fourth harmonic is dominant on the entire range, besides its poor relative intensity when compared to second and sixth harmonics (around $6 \mathrm{~dB}$ ).

(a)

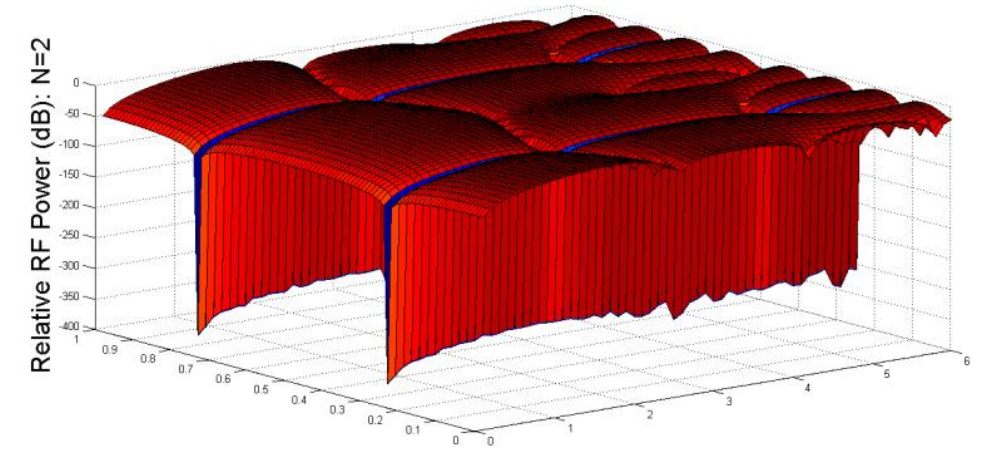

Normalized $\Gamma$ Parameter Modulation Index $\mathrm{m}$

(b)

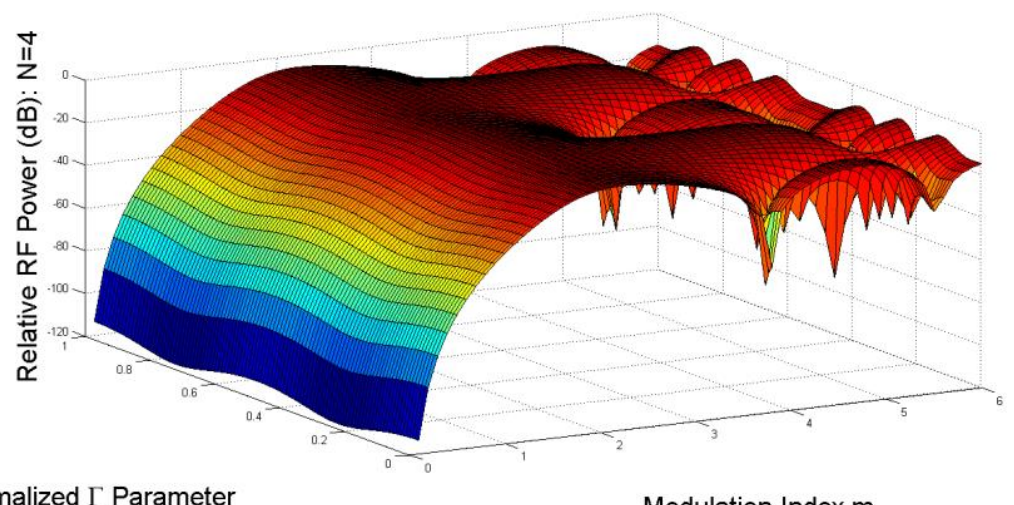

Normalized $\Gamma$ Parameter

Modulation Index m

Fig. 7. Amplitude of harmonics generated in a DD-MZM up-converter in a fiber optical link set for DSB modulation and MATB bias: (a) $2^{\text {nd }}$ and (b) $4^{\text {th }}$ harmonic dependence with respect to $\Gamma$ parameter and modulation index $m$.

(a)

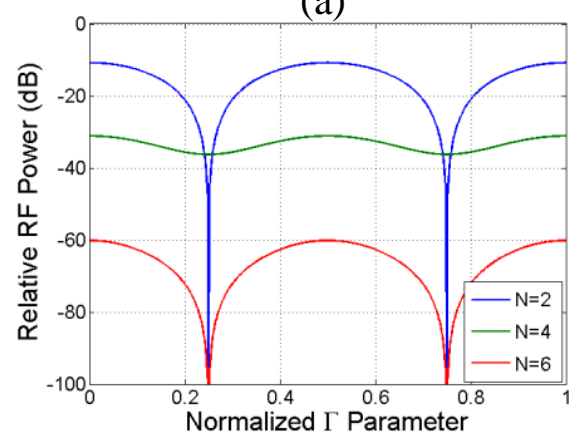

(b)

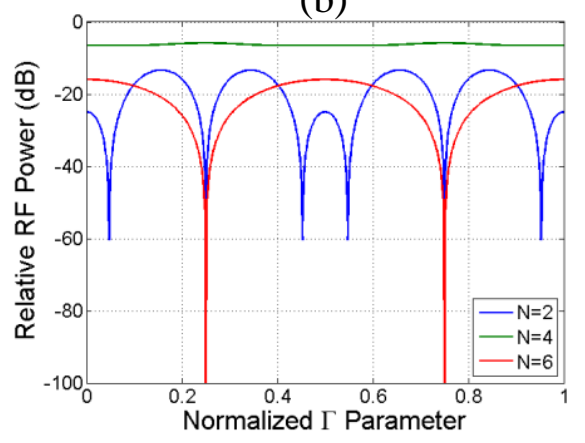

Fig. 8. Cuts in the amplitude 3D-plots of $2^{\text {nd }}, 4^{\text {th }}$ and $6^{\text {th }}$ harmonics for: (a) $m=1$ and (b) $=2.5$.

\section{EMPLOYMENT OF DP-MZM TO ENHANCE MULTIPLICATION FACTOR IN FIBER OPTIC LINKS}

Nowadays, innumerous applications in the area of telecommunication are being developed inside 
the $60 \mathrm{GHz}$ millimeter wave band $(57-64 \mathrm{GHz})$ [18]. In such frequency range it is widely recognized that the generation of high purity and low phase noise MMW signals using conventional electronic technology constitutes an expensive and challenging undertaking. As a consequence, in the past decade a great deal of research has been guided towards optical millimeter wave generation based on MZM's up conversion [6], [7], [8].

Signal generation in millimeter wave band using up-conversion techniques requires high multiplication factors. A photonic approach system based solely in DD-MZM is not adequate to reach that spectrum with easily available reference signals. Multiplication factors could be enhanced by associating DD-MZM in cascade and/or in parallel. Several topologies have being described in literature. While the simple ones control the basic parameters of the modulators in the system, complex configurations could employ Fiber Bragg Gratings (FBG), Erbium Doped Fiber Amplification (EDFA), Semiconductor Optical Amplifier (SOA) or other electrical or optical devices [3]. The main goal is trying to suppress certain optical spectral components, resulting more clearance between RF harmonics at the output. As mentioned before, this natural spacing reduces the need of optical filters and improves system performance. Among such experiments, steams out those dealing with frequency quadrupling based on an integrated dual parallel MZM (DP-MZM) [6], [7], [8]. The device's main configuration comprises three sub-MZM assembled as illustrated in

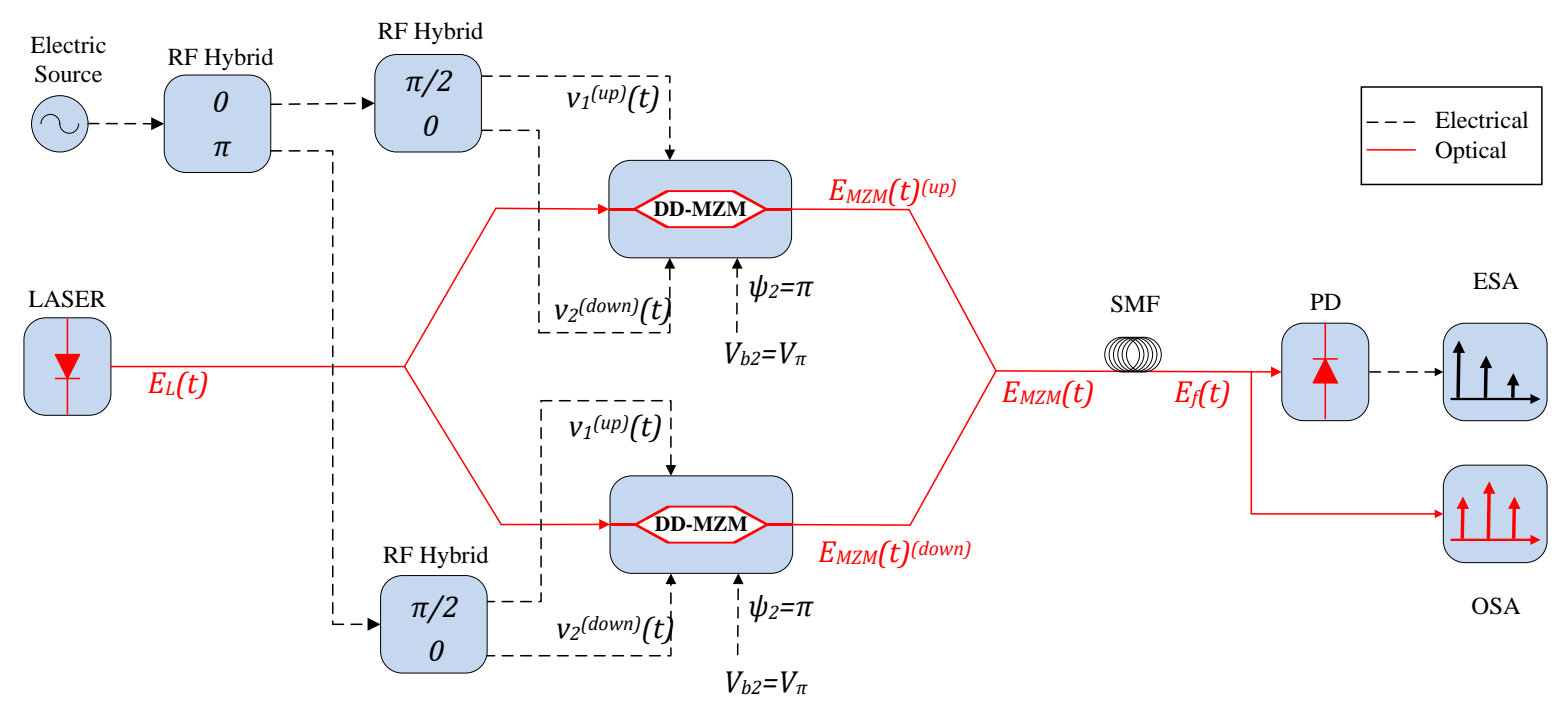

Fig. 9. The DD-MZM embedded in each arm of the main MZM exhibits features which were fully discussed in Section II. 


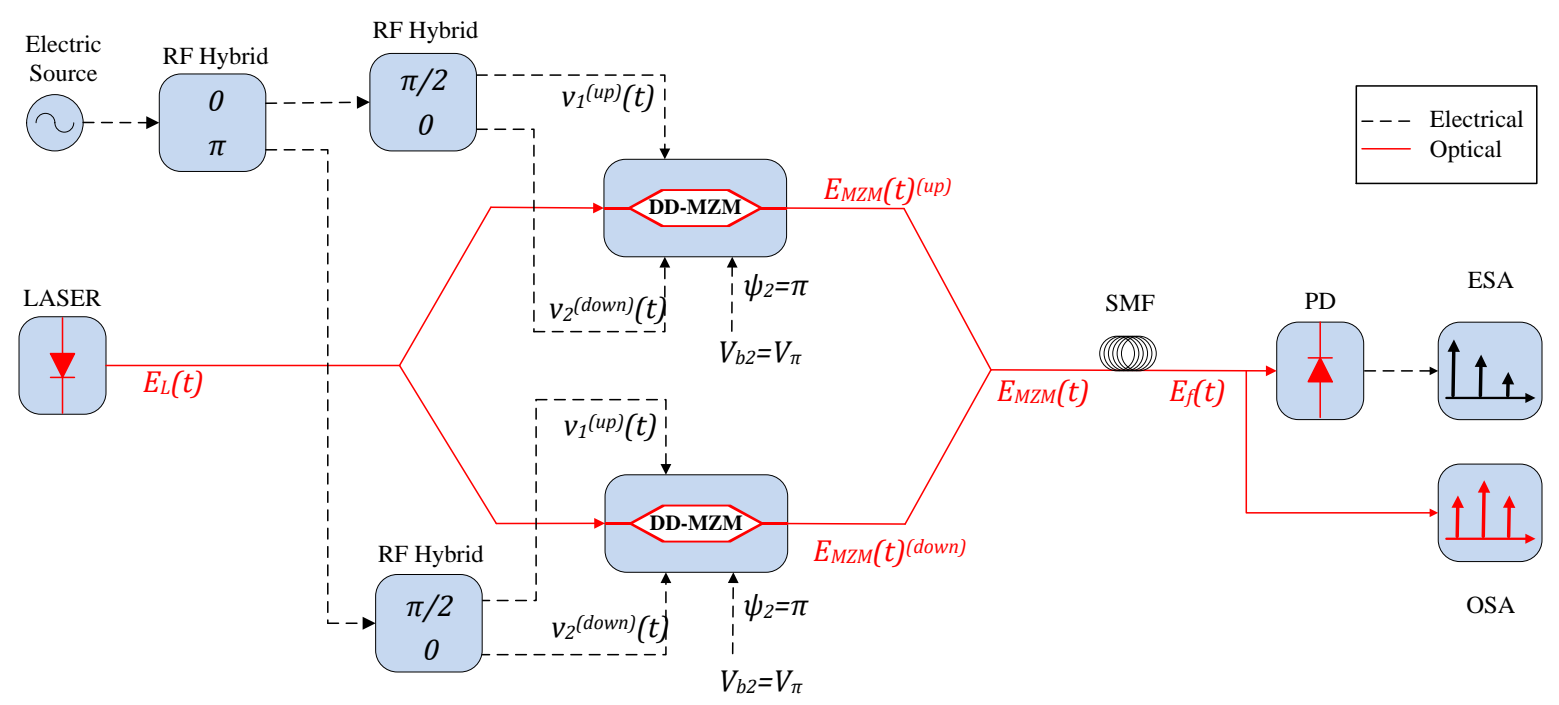

Fig. 9. Schematic representation of Dual-Parallel MZM (DP-MZM) in a fiber optical link (DD-MZM: Dual Drive Mach-

Zehnder Modulator; SMF: Single Mode Fiber; PD: Photodetector; OSA: Optical Spectrum Analyzer; ESA: Electrical Spectrum Analyzer).

To the best of our knowledge, the design of frequency quadrupler based on DP-MZM has been developed relying on models which does not fully takes into account the MZM's RF nonlinearity. For example, one of our paper main motivation is to examine the assumption which claims the existence of a certain modulation index depth beyond which no closed mathematical expression can be obtained for the RF output power [6], [7], [8]. To this aim, we represent the electric field at the DP-MZM output as the sum of the individual electric field from each DD-MZM (up and down):

$$
E_{M Z M}^{D P}(t)=E_{M Z M}^{D D}(t)^{(u p)}+E_{M Z M}^{D D}(t)^{(\text {down })}
$$

where

$$
\begin{gathered}
E_{M Z M}^{D D}(t)^{(u p)}=\frac{E_{0}}{4} e^{j \omega_{0} t}\left\{e^{j\left[m \cdot \cos \left(\omega_{R F} t+\pi / 2\right)\right]}+e^{j\left[m \cdot \cos \left(\omega_{R F} t\right)+\pi\right]}\right\} \\
E_{M Z M}^{D D}(t)^{(\text {down })}=\frac{E_{0}}{4} e^{j \omega_{0} t}\left\{e^{j\left[m \cdot \cos \left(\omega_{R F} t+3 \pi / 2\right)\right]}+e^{j\left[m \cdot \cos \left(\omega_{R F} t+\pi\right)+\pi\right]}\right\}
\end{gathered}
$$

After some algebraic operations, we get [9]:

$$
E_{M Z M}^{D P}(t)=-E_{0} e^{j \omega_{0} t} \sin \left[\frac{m}{\sqrt{2}} \sin \left(\omega_{R F} t+\frac{\pi}{4}\right)\right] \cdot \sin \left[\frac{m}{\sqrt{2}} \operatorname{sen}\left(\omega_{R F} t-\frac{\pi}{4}\right)\right]
$$

As expected, the RF nonlinearity effects on the optical field at DP-MZM's output, as given by (37), seems to be far more interesting than that previously investigated in Section II, which deals with DDMZM. In order to properly understand this statement one should apply the Jacobi-Anger expansion to rewrite (37) as a complex Fourier series:

$$
E_{M Z M}^{D P}(t)=\frac{E_{0}}{4} \sum_{n=-\infty}^{\infty} b_{n} e^{j\left(\omega_{0}+n \omega_{R F}\right) t}
$$

where 


$$
b_{n}=e^{j \frac{n \pi}{2}}\left(e^{j n \pi}+1\right)\left(e^{j \frac{n \pi}{2}}-1\right) J_{n}(m)
$$

Then, based in (39), it can be concluded that at the DP-MZM's output the optical carrier is fully suppressed. Beyond that, all the remaining $b_{n}$ coefficients are null, except those whose order obeys the relationship $n=4 k-2$, for $k=0, \pm 1, \pm 2, \pm 3 \ldots$. In another words, the optical spectrum only contains components at frequencies such as $\omega_{0} \pm 2 \omega_{R F}, \omega_{0} \pm 6 \omega_{R F}, \omega_{0} \pm 10 \omega_{R F}$, and so on (Fig. 10a). By comparing these characteristic to those illustrated in Fig. 5a, which are attached to DDMZM, steams out one of the main reason why DP-MZM is better suited for frequency quadrupling.
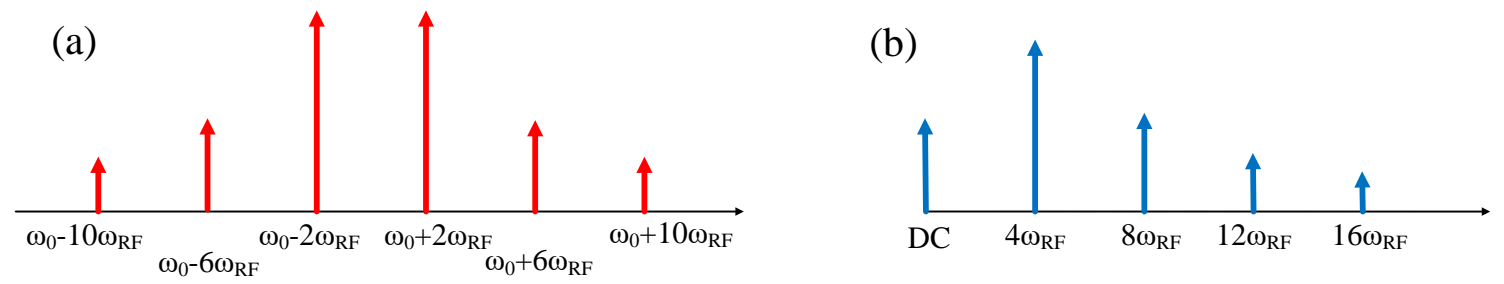

Fig. 10. Frequency response (the amplitude are not to scale) of a DP-MZM: (a) optical spectrum and (b) electronic spectrum.

Following the methodology described on previous section by considering a spool of fiber optic with length $L$, we obtain an expression for the exact current harmonic component at the photodetector output, in a similar way than (30):

$$
i\left(N \omega_{R F}\right)=\frac{1}{16} e^{j N \frac{\Gamma}{2}} \sum_{n=-\infty}^{\infty} b_{n+N} b_{n}^{*} e^{j n \Gamma}
$$

Plugging (39) in (40) and applying once again Graf's Addition Theorem, we have [10]:

$$
\begin{gathered}
i\left(N \omega_{R F}\right)=\frac{1}{16} e^{j \frac{N \pi}{2}}\left\{\left(1+e^{j \frac{N \pi}{2}}\right)\left(1+e^{j N \pi}\right)\right\}\left\{J_{N}\left[2 m \cdot \sin \left(\frac{\Gamma}{2}\right)\right]+J_{N}\left[2 m \cdot \cos \left(\frac{\Gamma}{2}\right)\right]\right. \\
\left.-e^{j \frac{N \pi}{4}}\left\{J_{N}\left[2 m \cdot \sin \left(\frac{\Gamma}{2}-\frac{\pi}{4}\right)\right]+J_{N}\left[2 m \cdot \sin \left(\frac{\Gamma}{2}+\frac{\pi}{4}\right)\right]\right\}\right\}
\end{gathered}
$$

The term $e^{j \frac{N \pi}{2}}$ is non-zero only when $N$ is a multiple of 4 . Therefore, the output electrical current will have components in the frequencies $4 \omega_{R F}, 8 \omega_{R F}, 12 \omega_{R F}$, and so on (Fig. 10b). Once again, harmonic electric current components depend on $\Gamma$ parameter and modulation index. The 3D plots of the fourth and eighth harmonics are presented in Fig. 11a and 11b. Cuttings on fixed $m$ allow identify the dominant harmonic and thus, the system multiplication factor. At $m=1$, Fig. 12a reveals that fourth harmonic is at least $74 \mathrm{~dB}$ higher than the eight harmonic, resulting in a high-purity signal with a large range insensibility to variations of link parameters. It is also noted a performance improvement when this DP-MZM quadrupling is compared to the DD-MZM doubling, by means of Fig. 12a and 8a: fading effect was very pronounced and then it is practically eliminated, for same modulation index. As an illustrative example, a $60 \mathrm{GHz}$ MMW signal could be generated from a $15 \mathrm{GHz}$ signal using this DP-MZM scheme, with less than $0.1 \mathrm{~dB}$ deviation with respect to ideal flat response of a standard single mode $80 \mathrm{~km}$ fiber optic link. This is a great length improvement when compared to 
the $1 \mathrm{~km}$ fading noticed in Fig. 6 [17]. Increases in modulation index will raise intensity of the other harmonics, as could be seen in Fig. 12b, for $m=5$.

(a)

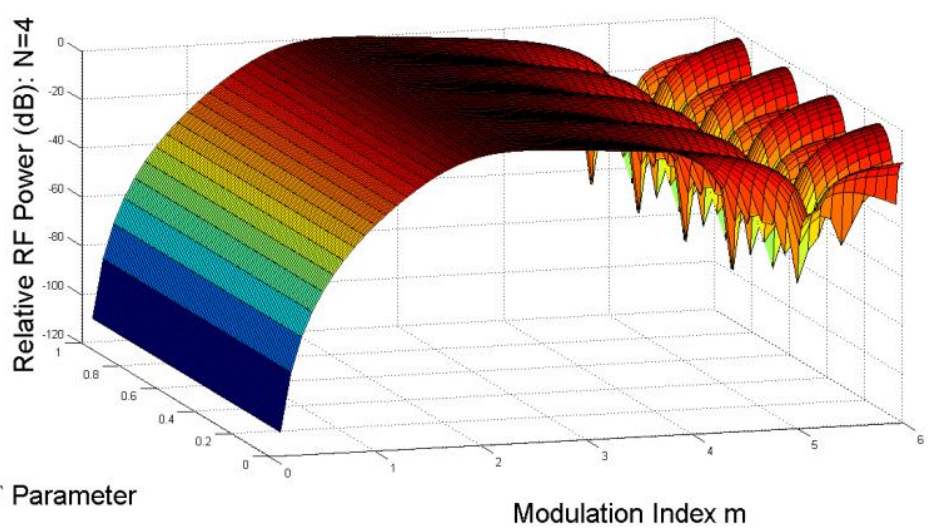

(b)

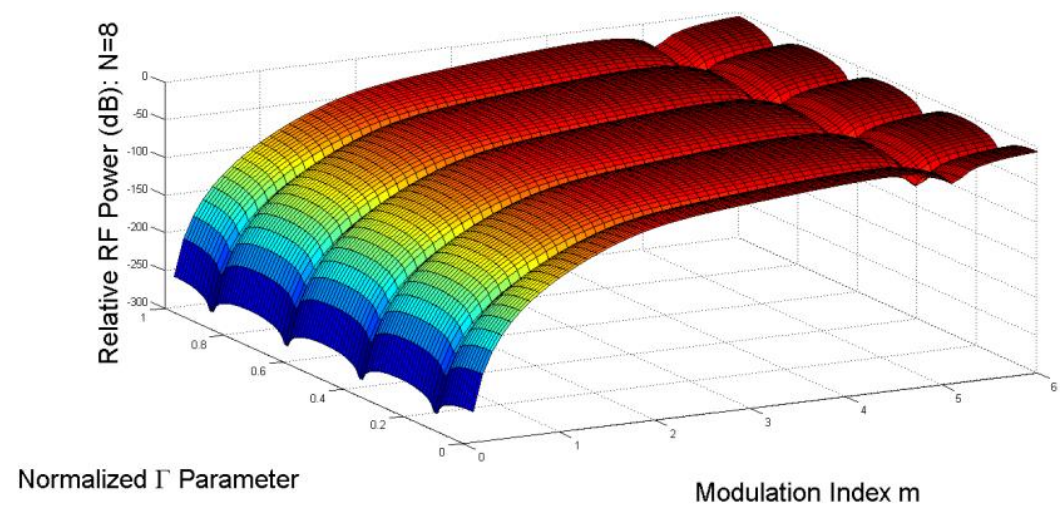

Fig. 11. Amplitude of harmonics generated in a DP-MZM up-converter in a fiber optical link: (a) $4^{\text {th }}$ and (b) $8^{\text {th }}$ harmonic dependence with respect to $\Gamma$ parameter and modulation index $m$.

(a)

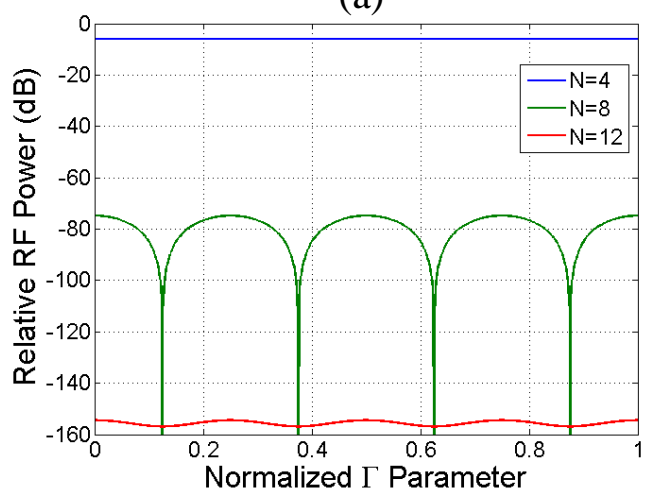

(b)

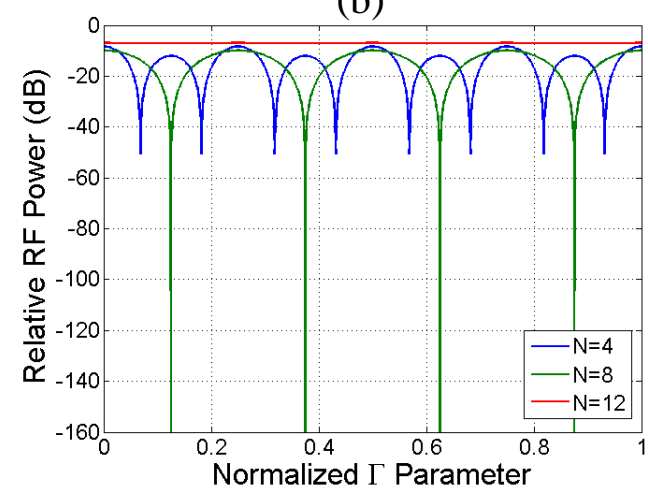

Fig. 12. Cuts in the amplitude 3D-plots of $4^{\text {th }}, 8^{\text {th }}$ and $12^{\text {th }}$ harmonics for: (a) $m=1$ and (b) $m=5$.

\section{DUAL PARALLEL MZM FREQUENCY QUADRUPLING}

It is opportune to point out that a great deal of previously published results on quadrupling are based on time domain analysis which leads to expression for the optical field in the form of infinite series [6], [7], [8], [14]. This fact makes the calculations very cumbersome, even when one attempts to fully take into consideration the RF nonlinearity at moderated level of optical modulation [12], [13]. Due to that, calculations are performed under the assumption that the series has a finite number of terms. Although a few approximations have been widely used, still there is the need for a more 
rigorous criterion that leads to an optimum value for the number of terms of the series.

In this publication we tackled the matter above mentioned using the theoretical background described in the previous section. Setting $\Gamma=0$ in (41), we exclude fiber optic from the system and (41) resumes to an exact and very concise expression for the current components at the PD's output [9]:

$$
i\left(N \omega_{R F}\right)=\left\{\begin{array}{cl}
\frac{1}{8}\left[J_{N}(0)+J_{N}(2 m)-2 e^{j \frac{N \pi}{4}} J_{N}(\sqrt{2} m)\right] & , \text { for } N=0,4,8,12 \ldots \\
0 & , \text { otherwhise }
\end{array}\right.
$$

First of all, we emphasize that (41) and (42) do not relay in any assumptions about the optical modulation index. Furthermore, they point out that the RF current only has spectral components at certain given frequencies, namely: $4 \omega_{R F}, 8 \omega_{R F}, 12 \omega_{R F}$, and so on. Such predictions are in full agreement with previously published values [6], [7], [8]. Moreover, the rather wide frequency range between two consecutives spectral components, which is twice as larger than that previously presented for the DD-MZM setup, constitutes one of the reasons why the DP-MZM configuration has become attractive as frequency up-converter. Regarding the magnitude of the already mentioned spectral components, their approximated expressions, presented by different authors, and the exact one, (42), are shown in Table II.

TABLE II. NORMALIZED RF CURRENT: DP-MZM (BTB)

\begin{tabular}{ccc}
\hline Harmonic & Exact Solution [9] & Approximated Solution [6], [7], [8] \\
\hline$i\left(4 \omega_{R F}\right)$ & $J_{4}(2 m)+2 J_{4}(\sqrt{2} m)$ & $J_{2}^{2}(m)+2 J_{2}(m) \cdot J_{6}(m)$ \\
$i\left(8 \omega_{R F}\right)$ & $J_{8}(2 m)-2 J_{8}(\sqrt{2} m)$ & $2 J_{2}(m) \cdot J_{6}(m)$ \\
$i\left(12 \omega_{R F}\right)$ & $J_{12}(2 m)+2 J_{12}(\sqrt{2} m)$ & $J_{6}^{2}(m)$ \\
\hline
\end{tabular}

Our numerical calculations regarding to the DP-MZM up-converter were constrained to BTB configuration, and were undertaken based on approximated and exact analytical expressions given in Table II. Since the exact expressions do not rely on any mathematical assumption with respect to the RF power drive, we developed the calculations for modulation index as high as 13 , as it is shown in Fig. 13. Actually, the various approximations introduced in [6], [7] and [8] still need a clear cut criterion to determine an upper bound limit for the modulation index.

(a)

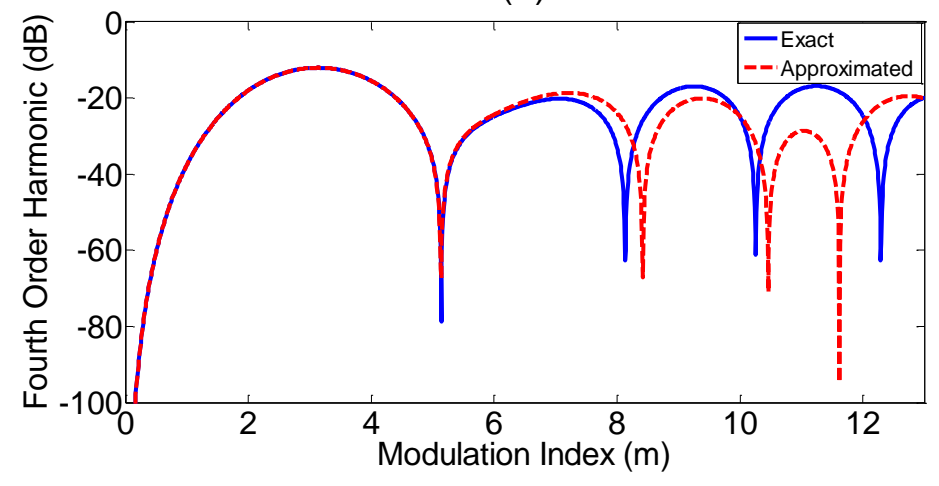


(b)

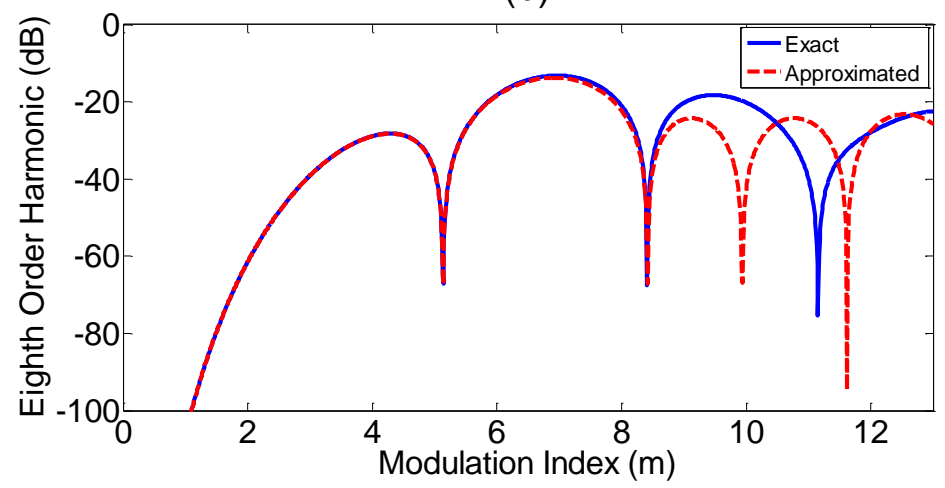

(c)

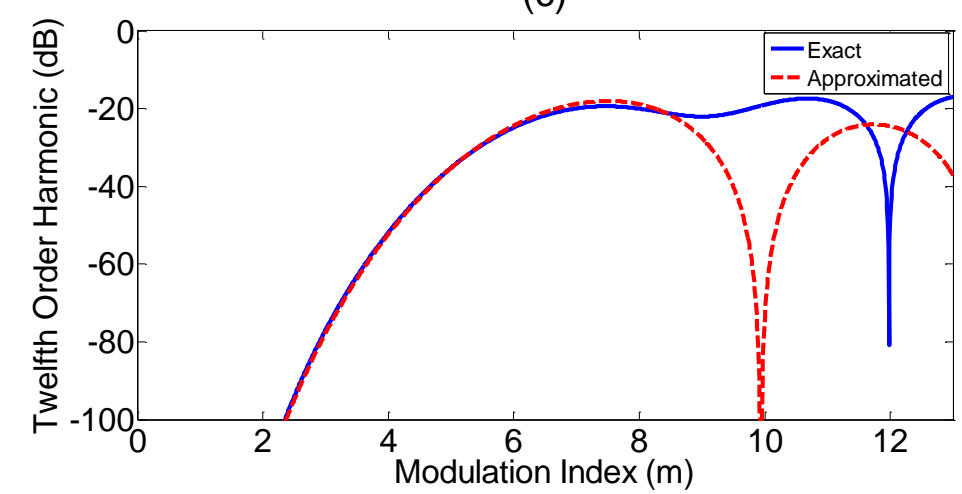

Fig. 13. DP-MZM's normalized RF power (BTB) according to Table II: (a) $4^{\text {th }}$ harmonic; (b) $8^{\text {th }}$ harmonic; (c) $12^{\text {th }}$ harmonic.

According to Fig. 13a, the two formulations are in very good agreement for modulation index as high as 5.0. For example, for $m=2.4$, the RF Sideband Suppression Ratio (RFSSR) using the exact and approximated relationships are found to be $36.18 \mathrm{~dB}$ and $36.10 \mathrm{~dB}$, respectively. The discrepancy between the two predictions only becomes relevant when the index exceeds 7.0. These results reveal that the limit of validation of the approximation lies far beyond 2.4 , as have been previously stated [6], [7], [8]. Similarly, the comparison between higher order harmonics amplitudes predicted by the two formulations also exhibits very good agreement for modulation index even higher than 5.0, as shown in Fig. 6b and Fig. 6c.

\section{CONCLUSION}

Wireless networks and radar systems are some of the major technological applications that rely on microwave and millimeter wave carrier signals. Photonic-based frequency multiplication topologies show up as tunable and reliable techniques. This paper presented the effects of chromatic dispersion in fiber optic links associated with two Mach-Zehnder Modulators in parallel, the so-called DualParallel MZM, to remotely provide microwave and millimeter wave signals from lower frequency reference signals. It is suitable to suppress certain optical spectral components through exploring the nonlinear characteristic of the external modulators. The result is sufficiently spaced RF components without using narrow optical or electrical filters. Analytical exact solution for the photodetector output current was derived with no restriction to small signal analysis. It allows a more accurate 
project design, once harmonic intensities could be precisely described by means of link parameters and modulation index. It has been proved that an up-conversion remote system can be achieved without considerable fading penalties due to fiber dispersion, by properly choosing link parameters.

Contrary to previously published formulations, which rely on time domain analysis, the analysis here presented was carried out in the frequency domain and yielded close expressions for the RF power at the DP-MZM's output. The obtained results reveal that previously published formulations do not properly take into account the RF nonlinearity. Thereby, one of the main advantages of the formulation is the fact that it enables straightforward calculations of parameters such as RFSSR, which otherwise has been only approximately calculated based on a wide range of methods. Phase noise and fiber nonlinearities effects are the next topic to be considered in the proposed model, along with practical experiments on future research.

\section{APPENDIX}

Nowadays, broadband travelling-wave electro-optic modulator based on lithium niobate $\left(\mathrm{LiNbO}_{3}\right)$, like MZM modulator, still play a major rule in high-speed and long-haul state-of-the art optical fiber communication networks. It is well known that RF frequency response of this type of modulator strongly depends on parameters such as the RF reflection coefficients, mismatching between optical and RF velocity, and electrodes losses, among others [19]-[24]. Quite often one assumes that the mentioned constraints are simultaneously overcome, and reaches the rather awkward conclusion that the link performance does not depend on the modulator bandwidth.

This appendix attempts to review models which accounts for effect of the previously identified mismatching issues on the modulator RF performance, based on unified nomenclature which enables a ready tracing back of results published by many authors. Before undertaking the analysis, we would like to point out that $\mathrm{LiNbO}_{3}$ is a dispersive material which exhibits an inherent mismatch between optical and microwave velocities, whereas the microwave attenuation and impedance mismatching are strongly dependents on electrodes geometries. Bearing in mind that a MZM modulator is made up of phase modulators (PM) properly connected, our model relies on the standard schematic representation shown in Fig. A-a. In such illustration, $v_{M}(t)$ stands for the RF drive voltage at the electrodes input, $Z_{M}$ is the internal impedance of signal generator, $Z_{L}$ is the load impedance at the electrodes right end, and $\mathrm{SiO}_{2}$ is the buffer layer. It is well known that the travelling-wave structures under investigation can support a quasi TEM mode, which can be represented by an equivalent transmission line, as shown in Fig. A-b. 


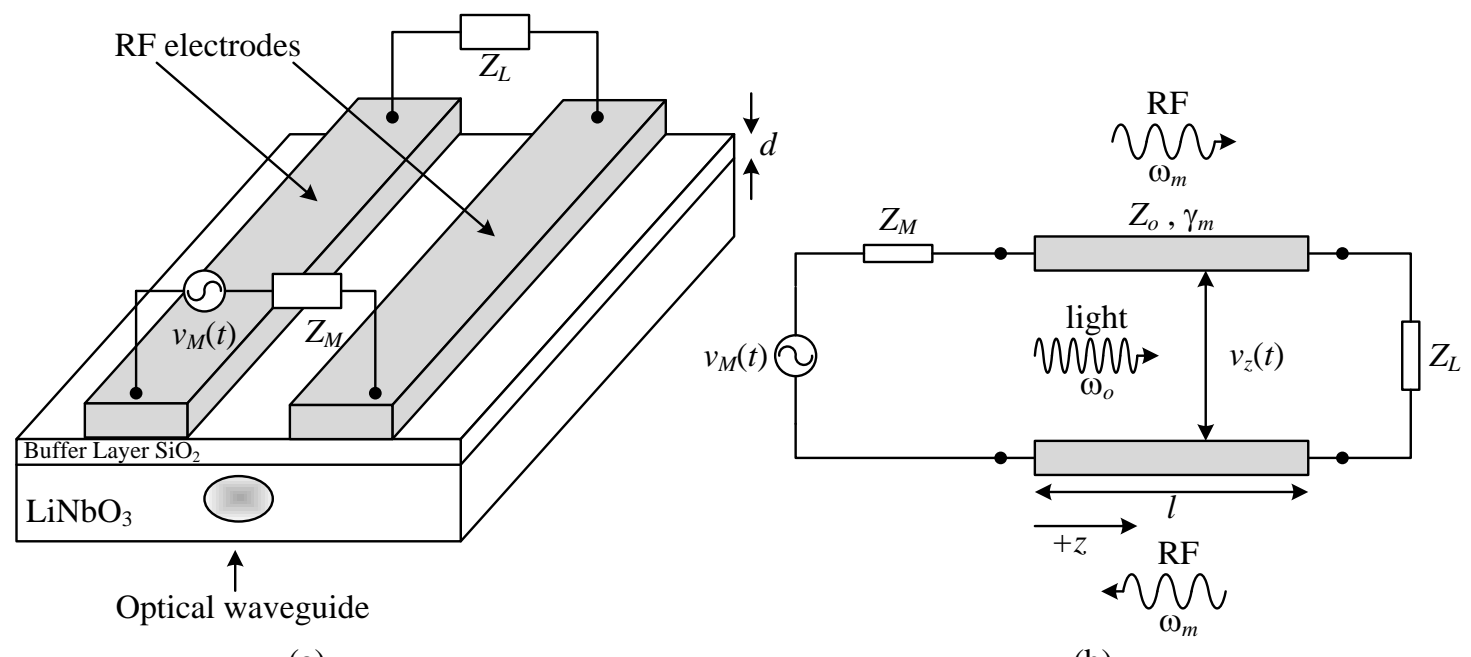

(a)

(b)

Fig. A. (a) Schematic representation of a LiNbO3 phase modulator with travelling-wave type electrodes. (b) Transmission line equivalent circuit of modulator electrodes.

Relying on classical electromagnetic theory to account the interaction between the RF and optical signal, through linear electro-optic effect (Pockel's effect), in combination with standard transmission line model one concludes that the RF voltage seen by the optical signal at position $z$ is given by:

$$
v\left(z, t_{0}\right)=\operatorname{Re}\left\{\frac{V_{M}}{2} \frac{\left(1-\Gamma_{g}\right)}{\left(1-\Gamma_{g} \Gamma_{L} e^{-2 \gamma_{m} l}\right)}\left[e^{-\alpha_{m} z} e^{j\left(\omega_{m} t_{0}-\xi_{i} \beta_{m} z\right)}+\Gamma_{L} e^{\alpha_{m} z} e^{-2 \gamma_{m} l} e^{j\left(\omega_{m} t_{0}+\xi_{r} \beta_{m} z\right)}\right]\right\}
$$

where $t_{o}$ is the instant at which the RF signal arrives at the interaction region, $V_{M}$ is the RF generator Thevenin's voltage, $\Gamma_{g}$ and $\Gamma_{L}$ are the reflection coefficients of generator and load, respectively; $\gamma_{m}$, $\alpha_{m}$ and $\beta_{m}$ are, respectively, the propagation, attenuation and phase factors; $l$ is the total interaction length; and $\omega_{m}$ is the angular frequency of the electrical signal.

An analysis of (A-1) reveals that its right hand side comprises two distinct parcels which are due to co-propagating $\left(-\beta_{m}\right)$, and counter-propagating $\left(+\beta_{m}\right)$ interactions between the optical and the RF signals. It should be noticed that the counter-propagating interaction steams out from the load impedance mismatching $\left(\Gamma_{L} \neq 0\right)$. The coefficients associated to phase velocity mismatching for the co-propagating $\left(\xi_{i}\right)$ and counter-propagating $\left(\xi_{r}\right)$ are given by:

$$
\begin{aligned}
& \xi_{i}=1-\frac{n_{o}}{n_{m}}=1-\frac{v_{m}}{v_{o}} \\
& \xi_{r}=1+\frac{n_{o}}{n_{m}}=1+\frac{v_{m}}{v_{o}}
\end{aligned}
$$

where $v_{m}$ and $v_{o}$ are the velocities of the electrical and optical signals, respectively; and $n_{m}$ and $n_{o}$ are the RF and optical index of refraction, respectively.

In summary, through (A-1) one can model the voltage along the PM electro-optic interaction length, taking into account the following parameters: the impedance mismatch, the RF and optical velocity mismatch, and the electrodes losses. Bearing in mind that a DD-MZM comprises four PM properly connected, it is also concluded that (A-1) can be used to modeling the voltage along each arm of such 
modulator.

Based on such assumptions, the total phase variation experienced by the optical wave after propagating through the interaction region, considering a drive signal in $t=t_{0}$, can be expressed as [25], [26]:

$$
\Delta \phi\left(t_{0}\right)=-\int_{0}^{l} \frac{\pi n_{o}^{3} r \Gamma_{\text {overlap }}}{G \lambda_{0}} v\left(z, t_{0}\right) d z
$$

where $r$ is the effective electro-optic coefficient, $G$ is the spatial gap between the electrodes, $\Gamma_{\text {overlap }}$ is the overlap integral between the microwave and optical fields [27], [28], and $\lambda_{0}$ is the optical wavelength.

In this publication, we emphasize DD-MZM having lossless electrodes $\left(\alpha_{m}=0\right)$ and a perfect impedance matching $\left(\Gamma_{g}=\Gamma_{L}=0\right)$. Hence, (A-4) reduces to:

$$
\Delta \phi\left(t_{0}\right)=\frac{\Delta \beta_{0} l}{2}\left[\operatorname{sinc}\left(\frac{\xi_{i} \beta_{m} l}{2}\right) \cos \left(\omega_{m} t_{0}-\frac{\xi_{i} \beta_{m} l}{2}\right)\right]
$$

where $\Delta \beta_{0}=\frac{-\pi n_{o}^{3} r \Gamma_{\text {overlap }} V_{M}}{G \lambda_{0}}$. Equation (A-5) is in agreement with previous publications [23], [29]. The PM performance analysis carried out using (A-5) shows that its bandwidth is limited by the phase velocity mismatching parameter. Now, if we consider a perfect velocity matching $\left(v_{m}=v_{o}\right)$ between microwave and optical waves, that results in $\xi_{i}=\xi_{r}=0$, (A-5) simplifies to:

$$
\Delta \phi\left(t_{0}\right)=\frac{\Delta \beta_{0} l}{2} \cos \left(\omega_{m} t_{0}\right)
$$

It should be pointed out that this approximate result has been widely used in the literature without the above given considerations. Such approach has been adopted in our analysis and leads to modulation index as given by (6). The voltage peak value that produces a phase change of $\pi$ radians in (A-6) is called half-wave switching voltage $\left(V_{\pi}\right)$. However, a general expression can be obtained by using (A-4). In this case, $V_{\pi}$ has dependence with frequency, conductor loss, interaction length, impedance mismatch, velocity mismatch, beyond the characteristics of manufacturing and materials of the modulator. Impedance mismatch causes microwave reflections in the device and results in a signal distortion for high-speed modulations [19]-[23]. Some design considerations to achieve broadband travelling-wave modulators are microwave and optical velocity match, low microwave electrode losses, low half-wave voltage $V_{\pi}$, and impedance matching. Tradeoffs are necessary due to the difficult to simultaneously satisfy all these requirements.

\section{ACKNOWLEDGMENT}

Authors would like to thanks Brazilian Air Force for the support and Electronic Warfare Laboratory at Technological Institute of Aeronautics for the facilities.

\section{REFERENCES}

[1] K. C. Huang, Z. Wang, "Millimeter wave communication systems," John Wiley \& Sons, 2011. 
[2] J. E. B. Oliveira; F. D. P. Alves; A. L. P. Mattei, "Trends in photonics applied to electronics warfare at Brazilian airforce," Proceedings of International Conference On Microwave And Optoelectronics, Rio de Janeiro: IEEE, 1999. p. 599-602.

[3] J. Yao, "A tutorial on microwave photonics," IEEE Photonics Society Newsletter, vol. 26, no. 2, pp. 4-12, April 2012.

[4] A. Kumar, V. Priye, "Photonic generation of microwave signal using a dual-parallel dual-drive Mach-Zehnder modulator," Proceedings of International Conference On Fiber Optics And Photonics, Kharagpur, 12, 2014.

[5] Y. Zhang, S. Pan, "Experimental demonstration of frequency-octupled millimeter-wave signal generation based on a dual-parallel Mach-Zehnder modulator," Proceedings of International Microwave Workshop Series On Millimeter Wave Wireless Technology And Applications, Nanjing, 2012.

[6] C-T. Lin, et al., "Optical millimeter-wave up-conversion employing frequency quadrupling without optical filtering," IEEE Transactions on Microwave Theory and Techniques, vol. 57, no. 8, pp. 2084-2092, Aug. 2009.

[7] C-T. Lin, et al., "Optical millimeter-wave signal generation using frequency quadrupling and no-optical filtering," IEEE Photonics Technology Letters, vol. 20, no. 12, pp. 1027-1029, June 2008.

[8] N. A. Al-Shareefi, et al., "Development of a new approach for high-quality quadrupling frequency optical millimeterwave signal generation without optical filtering," PIERS, vol. 134, pp. 189-208, 2013.

[9] A. A. Ferreira, J. A. Ribeiro, R. H. Souza, N. A. Roso, O. L. Coutinho, J. E. B. Oliveira, "Large signal analysis of photonic-assisted millimeter wave upconversion using Mach-Zehnder modulators," Anais do MOMAG 2014, Curitiba, p. 518-523 (2014).

[10] R. H. Souza, O. L. Coutinho, J. E. B. Oliveira, A. A. Ferreira, J. A. Ribeiro, "Effect of Fiber Optic Dispersion on Photonic Generated Millimeter Wave Using Dual-Parallel Mach-Zehnder Modulators," Anais do MOMAG 2016, Porto Alegre, 2016.

[11] Y. Shi, L. Yan, A.E. Willner, "High-speed electrooptic modulator characterization using optical spectrum analysis," IEEE Journal of Lightwave Technology, vol. 21, no. 10, pp. 2358-2367, October 2003.

[12] L. Cheng, S. Aditya, A. Nirmalathas, "An exact analytical model for dispersive transmission in microwave fiber-optic links using mach-zehnder external modulator," IEEE Photonics Technologies Letters, vol. 17, no. 7, pp. 1525-1527, July 2005.

[13] A. A. Ferreira, et al., "Effect of fiber optic chromatic dispersion on the performance of analog optical link with external modulation aiming at aerospace applications," Journal of Aerospace Technology and Management, vol. 5, no. 2, pp. 205-216, April 2013.

[14] H. Chi, X. Zou, J. P. Yao, "Analytical models for phase-modulation-based microwave photonic systems with phase modulation to intensity modulation conversion using a dispersive device," IEEE Journal of Lightwave Technology, vol. 27, no. 5, pp. 511-521, March 2009.

[15] G. P. Agrawal, "Nonlinear fiber optics," 5th ed., New York, Academic Press, 2013.

[16] H. Schmuck, "Comparison of optical millimetre-wave system concepts with regard to chromatic dispersion," Electronic Letters, v. 31, n. 21, p. 1848-1849, Oct. 1995.

[17] U. Gliese, S. Norskov, T. Nielsen, "Chromatic dispersion in fiber-optic microwave and millimeter-wave links," IEEE Trans. on Microwave Theory and Techniques, v. 44, n. 10, p. 1716-1724, Oct. 1996.

[18] S.Yu, W. Gu, A. Yang, T. Jiang, C. Wang, "A frequency quadrupling optical mm-wave generation for hybrid fiberwireless systems," IEEE Journal on Selected Areas in Communications/Supplement-Part 2, vol. 31, no. 12, December 2013.

[19] W. Yao, G. Giliard, M. K. Smit, M. J. Wale, "Equivalent Circuit Modelling of Integrated Traveling-Wave Optical Modulator in InP Foundry Platform," Proceedings of $18^{\text {th }}$ European Conference on Integrated Optics, Warsaw, 2016.

[20] O. Mitomi, K. Noguchi, H. Miyazawa, "Broadband and low driving-voltage $\mathrm{LiNbO}_{3}$ optical modulators," IEE Proc. Optoelectron., vol. 145, no. 6, pp. 360-364, Dec. 1998.

[21] G. K. Gopalakrishnan, W. K. Burns, R. W. McElhanon, C. H. Bulmer, A. S. Greenblatt, "Performance and Modeling of Broadband $\mathrm{LiNbO}_{3}$ Traveling Wave Optical Intensity Modulators," Journal of Lightwave Technology, vol. 12, no. 10, pp. 1807-1819, Oct. 1994.

[22] K. W. Hui, K. S. Chiang, B. Wu, Z. H. Zhang, "Electrode Optimization for High-Speed Traveling-Wave Integrated Optic Modulators," Journal of Lightwave Technology, vol. 16, no. 2, pp. 232-238, Feb. 1998.

[23] R. C. Alferness, S. K. Korotky, E. A. J. Marcatili, "Velocity-Matching Techniques for Integrated Optic Traveling Wave Switch/Modulators," Journal of Quantum Electronics, vol. QE-20, no. 3, pp. 301-309, Mar. 1984.

[24] O. Mitomi, K. Noguchi, H. Miyazawa, "Estimation of frequency response for high-speed $\mathrm{LiNbO}_{3}$ optical modulators," IEE Proc. Optoelectron., vol. 146, no. 2, pp. 99-104, Apr. 1999.

[25] D. Janner, D. Tulli, M. Belmonte, V. Pruneri, "Waveguide electro-optic modulation in micro-engineered $\mathrm{LiNbO}_{3}$," Journal of Optics A: Pure and Applied Optics, vol. 10, pp. 1-6, 2008.

[26] D. Janner, M. Belmonte, V. Pruneri, "Tailoring the Electrooptic Response and Improving the Performance of Integrated $\mathrm{LiNbO}_{3}$ Modulators by Domain Engineering," Journal of Lightwave Technology, vol. 25, no. 9, pp. 2402-2409, Sep. 2007.

[27] J. A. J. Ribeiro, J. E. B. Oliveira, "Comportamento Dinâmico do Modulador Eletroóptico de Ondas Caminhantes," Revista Telecomunicações, vol. 1, no. 1, pp. 29-35, Ago. 1998.

[28] C. Kitano, J. E. B. Oliveira, "Dispositivos à Óptica Integrada para Aplicações em Telecomunicações," Revista Telecomunicações, vol. 3, no. 2, pp. 27-38, Dez. 2000.

[29] Y.-q. Lu, M. Xiao, G. Salamo, "Wide-bandwidth high-frequency electro-optic modulator based on periodically poled $\mathrm{LiNbO}_{3}$," Applied Physics Letters, vol. 78, no. 8, pp. 1035-1037, Feb. 2001. 